\title{
Design of Metaheuristic Optimization-Based Vascular Segmentation Techniques for Photoacoustic Images
}

\author{
Thavavel Vaiyapuri $\mathbb{D}^{1},{ }^{1}$ Ashit Kumar Dutta ${ }^{(D)},{ }^{2}$ Mohamed Yacin Sikkandar $(D){ }^{3}$ \\ Deepak Gupta $\mathbb{D},{ }^{4}$ Bader Alouffi $\mathbb{D},{ }^{5}$ Abdullah Alharbi, ${ }^{6}$ Hafiz Tayyab Rauf $\mathbb{D},{ }^{7}$ \\ and Seifedine Kadry ${ }^{8}{ }^{8}$
}

${ }^{1}$ College of Computer Engineering and Sciences, Prince Sattam Bin Abdulaziz University, Al-Kharj, Saudi Arabia

${ }^{2}$ Department of Computer Science and Information Systems, College of Applied Sciences, AlMaarefa University, Ad Diriyah, Riyadh 13713, Saudi Arabia

${ }^{3}$ Department of Medical Equipment Technology, College of Applied Medical Sciences, Majmaah University, Al Majmaah 11952, Saudi Arabia

${ }^{4}$ Department of Computer Science \& Engineering, Maharaja Agrasen Institute of Technology, Delhi, India

${ }^{5}$ Department of Computer Science, College of Computers and Information Technology, Taif University, P.O. Box 11099, Taif 21944, Saudi Arabia

${ }^{6}$ Department of Information Technology, College of Computers and Information Technology, Taif University, P.O. Box 11099, Taif 21944, Saudi Arabia

${ }^{7}$ Department of Computer Science, Faculty of Engineering \& Informatics, University of Bradford, Bradford, UK

${ }^{8}$ Faculty of Applied Computing and Technology, Noroff University College, Kristiansand, Norway

Correspondence should be addressed to Thavavel Vaiyapuri; t.thangam@psau.edu.sa

Received 4 October 2021; Revised 5 January 2022; Accepted 10 January 2022; Published 30 January 2022

Academic Editor: Yuvaraja Teekaraman

Copyright (C) 2022 Thavavel Vaiyapuri et al. This is an open access article distributed under the Creative Commons Attribution License, which permits unrestricted use, distribution, and reproduction in any medium, provided the original work is properly cited.

\begin{abstract}
Biomedical imaging technologies are designed to offer functional, anatomical, and molecular details related to the internal organs. Photoacoustic imaging (PAI) is becoming familiar among researchers and industrialists. The PAI is found useful in several applications of brain and cancer imaging such as prostate cancer, breast cancer, and ovarian cancer. At the same time, the vessel images hold important medical details which offer strategies for a qualified diagnosis. Recently developed image processing techniques can be employed to segment vessels. Since vessel segmentation on PAI is a difficult process, this paper employs metaheuristic optimization-based vascular segmentation techniques for PAI. The proposed model involves two distinct kinds of vessel segmentation approaches such as Shannon's entropy function (SEF) and multilevel Otsu thresholding (MLOT). Moreover, the threshold value and entropy function in the segmentation process are optimized using three metaheuristics such as the cuckoo search (CS), equilibrium optimizer (EO), and harmony search (HS) algorithms. A detailed experimental analysis is made on benchmark PAI dataset, and the results are inspected under varying aspects. The obtained results pointed out the supremacy of the presented model with a higher accuracy of $98.71 \%$.
\end{abstract}

\section{Introduction}

Photoacoustic tomography (PAT) is a kind of hybrid imaging approach which integrates the advantages of both ultrasonic and optical imaging. In PAT, ultrasonic waves are stimulated by the pulsed laser that has embodied both ultrasonic deep penetration and optical absorption contrast
[1]. Various real-time applications are being studied to demonstrate their great opportunities in both clinical and preclinical imaging, such as breast cancer diagnostics and small animal entire body imaging [2]. In addition, multispectral PAT has exclusive benefits from monitoring the functional data of biological tissues, for example, metabolism and blood oxygen saturation (sO2). Especially, 
photoacoustic computed tomography (PACT) allows realtime imaging performances that disclose great possibilities for medical application. In order to attain the image in the PA signal, the image reconstruction approach plays a major part. Traditional noniterative reconstruction approaches, such as filtered back-projection (FBP), are widespread because of their fast speed. But, the imperfection delay-andsum (DAS) of traditional approaches exist several artifacts that lead to distorted images, particularly in restricted view configuration [3-5]. In such cases, the iterative methods are well-adopted when using an appropriate regularization. PAT is a noninvasive combined physics biomedical imaging approach that conveniently integrates the higher contrast of pure optical imaging through the higher spatial resolution of ultrasound imaging [6]. It can be dependent upon the generation of acoustic waves through illuminating a semitransparent biological or clinical object with a shorter optical pulse. The induced time-based acoustic wave is measured outside of the samples using an acoustic detector, and the measured information is utilized for recovering an image of the interior [7].

Vessel segmentation is a fundamental process for analyzing biomedical imaging. Hence, it is complicated for providing accurate and reliable vessel segmentation on PA imaging. [8] shows better segmentation performance on the blood vessels taken from photoacoustic computed tomography (PACT). Optical resolution photoacoustic microscopy (OR-PAM) features simple imaging reconstruction and higher spatial resolution [9] and therefore has initiated broad application in vascular imaging. Up till now, several attempts have focused on the segmentation of the blood vessel taken by the OR-PAM method. Image segmentation has been considered an essential task in image processing and plays a significant part in image research in various areas of application such as pattern recognition, computer vision, medical imaging, robotic vision, cryptography, and agriculture [10]. When the results of segmentation are not correct, the classification approach might fail. Multiple challenges have been widely resolved by the segmentation method. The major purpose of segmentation is to split an image into many homogeneous segments/regions with common features such as color, texture, contrast, brightness, size, and form depending upon certain thresholding value(s) [11]. Often, it is extensively employed for distinguishing background and foreground as the primary phase for interpreting and identifying images.

Various methodologies, like threshold-based, regionbased, feature-based, and edge-based clustering, were proposed for solving present challenges and enhance quality of the research. The most popular image segmentation methods are multilevel thresholding that tries to group the pixel which allocates features to a finite amount of pixels. Image thresholding methods are easier to produce and implement efficient than segmentation performances [12]. Thresholding comprises 2 classes: bi and multilevel. A single threshold value is utilized in the preliminary class for separating the images into 2 homogeneous background and foreground regions. The latter methods are employed for segmenting an image into more than 2 areas according to the pixel intensity called a histogram. Therefore, it can be expressed as an optimization issue with parametric/nonparametric methods.

Since the vessel segmentation on PAI is a difficult process, this paper employs metaheuristic optimizationbased vascular segmentation techniques for PAI. This study employs two different types of vessel segmentation techniques such as Shannon's entropy function and Otsu thresholding. In addition, the threshold value and entropy function in the segmentation process are optimized using three metaheuristics such as the cuckoo search (CS), equilibrium optimizer (EO), and harmony search (HS) algorithms. For examining the improved performance of the proposed model, a wide range of simulations take place and the results are inspected under various aspects.

The rest of the paper is organized as follows: Section 2 offers the related works, Section 3 provides the proposed model, Section 4 gives the experimental validation, and Section 5 concludes the study.

\section{Literature Review}

Luke et al. [13] proposed a novel deep neural network (DNN) method for concurrently estimating the oxygen saturation in blood vessels and segmenting the vessels in the nearby background tissues. The network was trained on evaluating early pressure distribution from a 3D Monte Carlo simulation of light transport in breast tissues. Bench et al. [14] illustrate the capacity of DNN for processing entire three-dimensional images and outputting three-dimensional maps of vascular sO2 from realistic tissue images or models. The 2 separate fully convolutional neural network (FCNN) methods have been trained for producing three-dimensional maps of vascular blood oxygen saturation and vessel position from multiwavelength simulated images of tissue models.

In [15], a novel segmentation system is developed for PAI which directly produces an assessment of its consistency. Segmentation based on parameters has a natural tendency to improve consistency as the parameter value monotonically changes. The consistency is measured by calculating the classification of an image voxel with distinct parameters. In [16], U-net and FCN have been individually employed in PA imaging for vascular segmentation, and a hybrid network consists of both that is integrated by a voting system on the PA vascular image. The result is qualitatively related and estimated on intersection of union (IoU), dice coefficients, accuracy, and sensitivity.

In [17], tumor vessel is quantified and segmented in a whole three-dimensional framework. It has been tested from the phantom experiment that the three-dimensional quantification outcomes have higher performance when compared to two dimensions. Furthermore, in vivo vessel images have been quantified with two- and three-dimensional quantification systems correspondingly. Additionally, the variation between these 2 outcomes is important. Zhao et al. [18] presented an approach adoptive to the microvascular segmentation in photoacoustic image that includes morphological connection and a Hessian matrix 
enhancement operator. The precision of these vascular segmentation approaches is quantitatively estimated by several criteria. In order to attain continuous and accurate microvascular skeletons, an enhanced skeleton extractionbased multistencil fast marching (MSFM) approach has been presented.

Boink et al. [19] suggest conjointly obtaining the photoacoustic segmentation and reconstruction by altering a newly presented partially learned model based on the CNN method. They examine the stability of the method against modifications in early pressure and photoacoustic system settings. This insight is utilized for developing a method, i.e., strong input and system setting. This method could simply be employed in other imaging models and adapted to implement other higher-level processes dissimilar to segmentation. Feng et al. [20] proposed a vivo experiment and numerical simulation on human subjects for investigating the opportunity of segmentation of photoacoustic signals and ultrasound guided detection from bone tissues in vivo in a noninvasive method.

\section{Materials and Methods}

In this study, a set of metaheuristic optimization-based vascular segmentation techniques have been developed for PAI. The goal of the study is to inspect the outcome of the dissimilar metaheuristics on two segmentation approaches. In order to successfully segment the vessels, the SEF and MLOT techniques are applied. Furthermore, three optimization algorithms such as CS, EO, and HS are applied for optimal choice of entropy function and threshold values. Figure 1 demonstrates the overall block diagram of the proposed method.

3.1. Image Segmentation Approach. In this study, SEF and MLOT techniques were applied to segment the vessels on PAI. The detailed workings of these two segmentation approaches are provided in the following:

3.1.1. Design of Shannon's Entropy Function. Shannon's entropy model is an important concept from the field of "information theory and coding." This theory has been utilized for probabilistically determining the count of data broadcast with some data. Consider that an image is a $(k+$ 1) homogeneous area with a $k$ threshold gray level at $t_{1}, t_{2}, t_{3}, \ldots, t_{k}$, then the following equation is obtained:

$$
h(i)=\frac{f_{i}}{N}, \quad i=0,1,2,255,
$$

where $f_{i}$ represents the frequency of $i^{\text {th }}$ gray levels, $N$ implies the entire amount of gray levels existing from the images, and $h(i)$ refers the normalization frequency. The Shannon entropy function has been defined as follows [21]:

$H=-\sum_{i=0}^{t_{1}} P_{1 i} \ln \left(P_{1 i}\right)-\sum_{i=t_{1}+1}^{t_{2}} P_{2 i} \ln \left(P_{2 i}\right)-\cdots-\sum_{i=t_{k}}^{255} P_{k i} \ln \left(P_{k i}\right)$, where

$$
\begin{aligned}
& P_{1 i}=\frac{h(i)}{\sum_{i=0}^{t_{1}} h(i)}, \quad \text { for } 0 \leq i \leq t_{1}, \\
& P_{2 i}=\frac{h(i)}{\sum_{i=t_{1}+1}^{t_{2}} h(i)}, \quad \text { for } t_{1}+1 \leq i \leq t_{2}, \\
& P_{k i}=\frac{h(i)}{\sum_{i=t_{k}+1}^{255} h(i)}, \quad \text { for } t_{k}+1 \leq i \leq 255 .
\end{aligned}
$$

The Shannon entropy function $H$ from equation (1) has been executed as an objective function that can be maximized using the metaheuristic optimization algorithms such as CS, HS, and $\mathrm{EO}$ algorithms.

3.1.2. Design of Multilevel Ostu Thresholding (MLOT) Technique. Otsu [22] is a widely employed segmentation approach utilized for finding a better threshold value of an image dependent upon maximizing the between-class variance. These techniques were utilized for finding the threshold optimal value which split the image into several classes. This technique recognizes the $L_{v}$ intensity level of gray images and the probability distribution has been estimated in equation (2). It could be utilized to color images, in that Otsu has been implemented to all channels.

$$
\begin{aligned}
h_{i} & =\frac{h_{i}}{N P}, \\
\sum_{i=1}^{N P} P h_{i} & =1,
\end{aligned}
$$

where $i_{l}$ refers the intensity level identified from the range of $\left(0 \leq i_{l} \leq L_{v}-1\right)$. NP signifies the entire amount of image pixels. $h_{i}$ indicates the amount of the presence of intensity $i_{l}$ under the image signified as the histograms. The histogram has been normalized from the probability distributions of $P h_{i}$. According to the probability distribution/thresholding value $(t h)$, the classes have been defined to bilevel segmentation as follows:

$C_{1}=\frac{P h_{1}}{\omega_{0}(t h)}, \ldots, \frac{P h_{t h}}{\omega_{0}(t h)}$ and $C_{2}=\frac{P h_{t h+1}^{c}}{\omega_{1}(t h)}, \ldots, \frac{P h_{L}}{\omega_{1}(t h)}$,

where $\omega_{0}(t h)$ and $\omega_{1}(t h)$ are cumulative probability distribution to $C_{1}$ and $C_{2}$, respectively, as it can be demonstrated in equation (4).

$$
\omega_{0}(t h)=\sum_{i=1}^{t h} P h_{i} \text { and } \omega_{1}(t h)=\sum_{t h+1}^{L} P h_{i} .
$$

It can be required for finding the average intensity levels $\mu_{0}$ and $\mu_{1}$ which are utilized in equation (5). If these values are $c$, the Otsu based on class $\sigma_{B}^{2}$ is determined in equation (6).

$$
\mu_{0}=\sum_{i=1}^{t h} \frac{i P h_{i}}{\omega_{0}(t h)} \text { and } \mu_{1}=\sum_{i=t h+1}^{L} \frac{i P h_{i}}{\omega_{1}(t h)}, \sigma_{B}^{2}=\sigma_{1}+\sigma_{2} .
$$




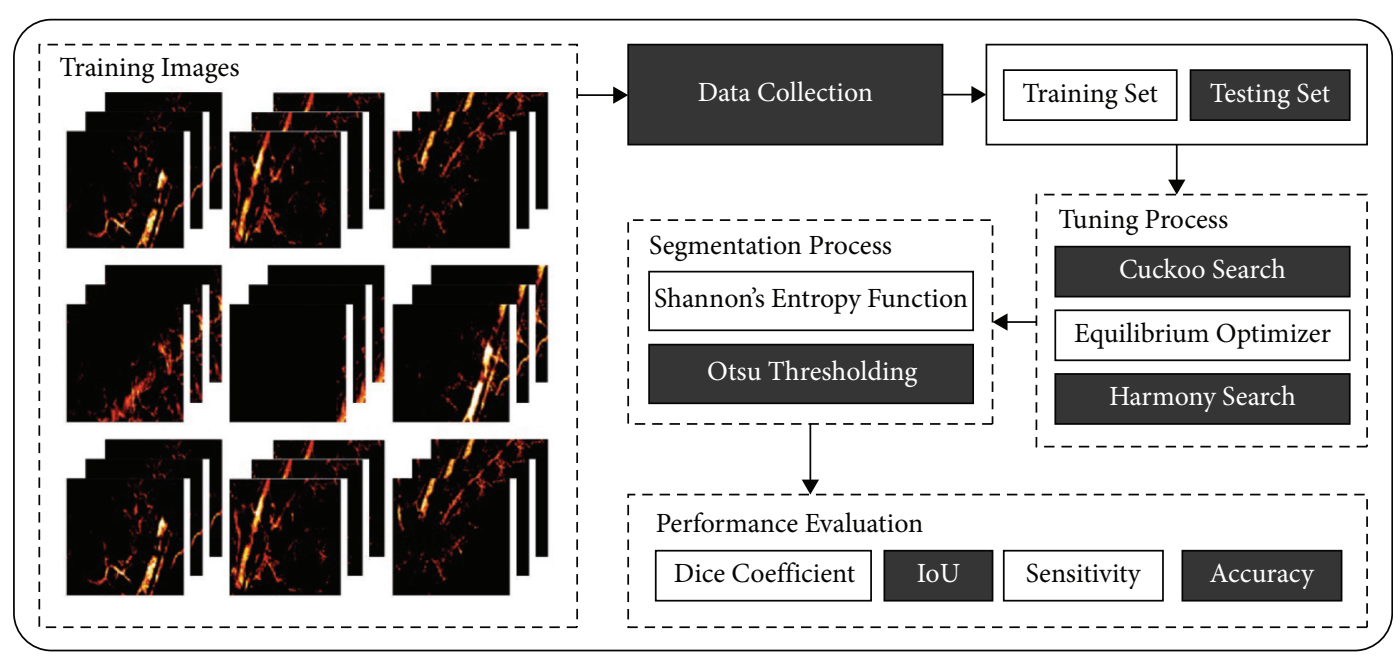

Figure 1: Overall process of the proposed method.

Noticeable $\sigma_{1}$ and $\sigma_{2}$ in equation (6) are the differences of $C_{1}$ and $C_{2}$ which are identified as follows:

$$
\sigma_{1}=\omega_{0}\left(\mu_{0}+\mu_{T}\right)^{2} \text { and } \sigma_{2}=\omega_{1}\left(\mu_{1}+\mu_{T}\right)^{2},
$$

where $\mu_{T}=\omega_{0} \mu_{0}+\omega_{1} \mu_{1}$ and $\omega_{0}+\omega_{1}=1$ are dependent upon the values $\sigma_{1}$ and $\sigma_{2}$, and equation (8) offers the objective functions. So, the optimized issue has been decreased for finding the intensity level that maximizes in equation (8).

$$
F_{\text {otsu }}(t h)=\max \left(\sigma_{B}^{2}(t h)\right) \text { where } 0 \leq t h \leq L-1,
$$

where $\sigma_{B}^{2}(t h)$ stands for Otsu's alteration to provided th value. An objective function $F_{\text {otsu }}(t h)$ in equation (8) is also altered to several thresholds as follows:

$$
\begin{aligned}
F_{\text {otsu }}(T H) & =\operatorname{Max} \sigma_{B}^{2}(t h) \text { where } 0 \leq t h_{i} \leq L-1 \text { an } d i \\
& =[1,2,3, \ldots, k](9),
\end{aligned}
$$

where $T H=\left[t h_{1}, t h_{2}, \ldots, t h_{k}-1\right]$ has been a vector comprising several thresholds, $L$ refers to the maximal gray level, but the alterations are computed in equation (10).

$$
\begin{aligned}
\sigma_{B}^{2} & =\sum_{i=1}^{k} \sigma_{i} \\
& =\sum_{i=1}^{k} \omega_{1}\left(\mu_{1}-\mu_{T}\right)^{2},
\end{aligned}
$$

where $i$ demonstrates the particular class, and $\omega_{i}$ and $\mu_{j}$ implies the probability of existence and the mean of level, respectively. Multilevel thresholding values are attained as follows:

$$
\omega_{k-1}(t h)=\sum_{i=t h_{k}+1}^{L} P h_{i}
$$

With respect to the mean values as defined in equation (12), the followingequationis obtained:

$$
\mu_{k-1}=\sum_{i=t h_{k}+1}^{L} \frac{i P h_{i}}{\omega_{1}\left(t h_{k}\right)} .
$$

In this study, the threshold values can be optimally chosen by the use of three optimization algorithms, namely, CS, HS, and EO algorithms.

3.2. Metaheuristic Optimization Models. In this section, the algorithmic procedures of the CS, EO, and HS algorithms are elaborated in detail. These algorithms are employed for the proficient selection of the entropy function in SEF and the threshold value in the MLOT technique.

3.2.1. Process Involved in the CS Algorithm. The CS technique is simulated by the social performances of cuckoos. The CS is a population-based search technique for identifying an optimum result of optimized issues. A novel set of experimental results utilizing this technique tries to determine, on previous initiation, the optimum experimental results. The general framework and competence to explain these issues with distinct execution can be carried out with the CS technique. The CS has been established in concern of the social pensive ability of the cuckoo bird. Generally, the cuckoo bird places its eggs from the nest of another alien bird. The alien bird is declaring that the egg has the associated probability of $p a \in[0,1]$. Therefore, it is also the egg that has been thrown in the nest or forsaken which nest to construct a novel one with the alien bird. Thus, all the eggs of the alien nest carried out a solution.

Mathematically, the CS algorithm is expressed in three major levels. Initially, the accessibility of the host nest was set. The next posit is that all cuckoos locate only a single egg at a timestamp for a randomly chosen host nest, and finally, the maximum quality eggs from the optimum nest are surplus to the next generations [23]. Assume that $X_{i}(t)$ implies the current search space of cuckoo iwherei $=1,2, \cdots, N$ at a time $t$, represented as $X_{i}=\left(x_{i}^{1}, x_{i}^{2}, \ldots \ldots, x_{i}^{n}\right)$ from the $n$ dimension quandary. Afterward, the initial search space $X_{i}(t+1)$ to the later generations at time $t+1$ has been mathematically computed as follows: 


$$
X_{i}(t+1)=X_{i}(t)+\alpha \operatorname{Lev} y(\lambda)
$$

where $\alpha>0$ represents the step-size that is similar to the scales of the quandary of the curiosity. In maximal cases $\alpha$ is generally taken as 1 .

It offers a random walk, and their random steps have been drawn in Levy distribution to immensely colossal steps that are demonstrated as follows:

$$
\text { Levy }(\lambda) u=t^{-\lambda}, \quad \text { where } 1<\lambda<3 \text {. }
$$

The 2 very famous techniques are Mantegna's technique and McCulloch's technique. The Levy step size has been attained in the Mantegna technique as initialized, which is as follows:

$$
\operatorname{Lev} y=\frac{v}{|\nu|^{1 /(\lambda-1)}} \text {. }
$$

The conditions for forsake probabilities, the entire size of populations, and the maximal number of cuckoos reproduced in a lifetime are fixed to the utilizer; however, primary terms at the starting has been reached. Figure 2 depicts the flowchart of the CS technique.

Assume that $t$ symbolizes the current generation, $t_{\max }$ signifies the determined greatest span of animating being life redundancy, and that the cuckoo influence roams from the lifetime. Therefore, an $n$-dimension issue to cuckoo at the starting generation $t=1$ is that set up and it can be represented in equation (16).

$$
x_{1}^{n}(t=1)=\operatorname{rand} n \times\left(\text { Upper }^{n}-\text { Lower }^{n}\right),
$$

where Lower ${ }^{n}$ and Upper $^{n}$ refer the lowermost as well as uppermost outer restricts of the search space of $n$th attributes, respectively. These maintain the mastery capable time-optimized technique to stay within the individual perimeter.

In equation (15), $v$ and $v$ demonstrate the taken in normal distributions. That means $v \sim N\left(0, \delta^{2}\right)$ and $\nu \sim N(0,1)$ with the following:

$$
\delta=\left(\frac{\Gamma(1+\beta) \sin (\pi \pi / 2)}{\Gamma(1+\beta / 2) \beta \times 2^{((\beta-1) / 2)}}\right)^{1 / \beta},
$$

where $\Gamma$ stands for the gamma function, and it can be demonstrated as follows:

$$
\Gamma(p)=\int_{0} e^{-t} t^{p-1} \mathrm{~d} t \& \beta \in[0,2] \text { is a scale factor. }
$$

3.2.2. Process Involved in the EO Algorithm. EO follows a dynamic mass balance process that works to control volume. The mathematical formula has been utilized for representing the mass balance for defining the focus of nonreactive components in the dynamic environments of control volume and this formula as a function with their different processes in the changes of source and sink. The entire theoretical explanation of EO according to these terms is defined as follows:

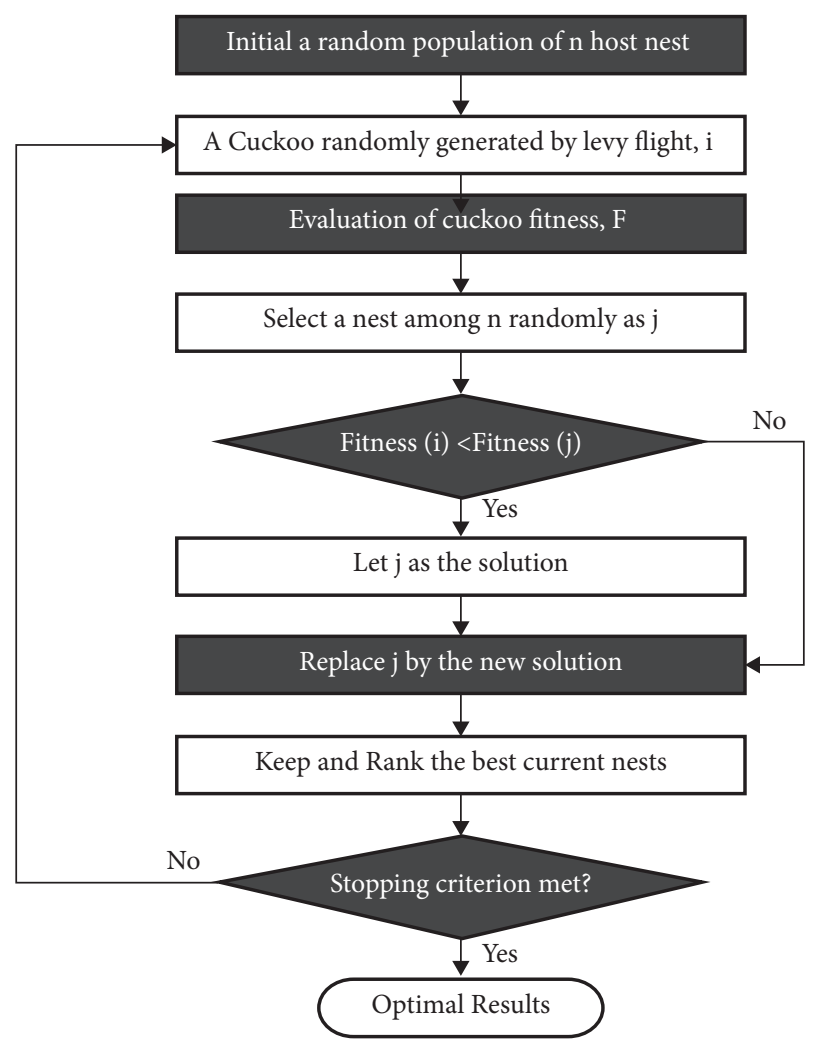

FIgURE 2: Flowchart of the CS algorithm.

The arbitrary population (primary concentration) was initialized by utilizing a uniform distribution dependent upon the amount of particles and dimension in the provided search region as follows:

$$
C_{i}^{\text {initial }}=C_{\min }+\operatorname{rand}_{i}\left(C_{\max }-C_{\min }\right) i=1,2, \ldots, n,
$$

where $C_{i}^{\text {initial }}$ represents the vector of primary concentration of ith particle, $C_{\min }$ and $C_{\max }$ refer the lower as well as upper bounds, rand $_{i}$ signifies the uniform arbitrary numbers created in intervals from 0 to 1 , and $n$ defines the size of populations.

In order to determine the equilibrium state (global optimum), a pool of 4 optimum candidates is identified, that comprises of particles with a concentration identical to arithmetic mean of 4 particles. These particles produced a pool vector, as ffgiven in (20).

$$
\vec{C}_{e q . p o o l}=\left\{\vec{C}_{e q(1)}, \vec{C}_{e q(2)}, \vec{C}_{e q(3)}, \vec{C}_{e q(4)}, \vec{C}_{e q(\text { ave })}\right\}
$$

In the evolution procedure, initial particles upgrade their concentration from the initial generation dependent upon $\vec{C}_{e q(1)}$, and then in the second generation, the upgrading can take place on $\vec{C}_{e q(\text { ave })}$. Afterward, all the particles with every candidate solution have been upgraded by the completion of the evolution procedure.

The exponential term $F$ demonstrates that it assists EO with attaining the appropriate balance amongst diversification as well as intensification [24]. The term $\lambda$ has been an 
arbitrary value from the intervals 0 and 1 for controlling the turnover rate from real control volume.

$$
\vec{F}=e^{-\vec{\lambda}\left(t-t_{0}\right)},
$$

where $t$ has been provided as function of the amount of iteration (Iter) and is determined as follows:

$$
t=\left(1-\frac{\text { Iter }}{\text { Max_iter }}\right)\left(a_{2} \frac{\text { Iter }}{\text { Max_iter }}\right),
$$

where Iter $=$ current iteration, Max_iter $=$ maximal iteration, and parameter $a_{2}$ was employed for controlling exploitation capability of EO. For ensuring convergence while improving global as well as local search capability of the technique, in written form is also employed as follows:

$$
\overrightarrow{t_{0}}=\frac{1}{\vec{\lambda}} \ln \left(-a_{1} \operatorname{sign}(\vec{r}-0.5)\left[1-e^{-\vec{\lambda} t}\right]\right)+t,
$$

where $a_{1}$ and $a_{2}$ have been utilized for controlling global as well as local search capability of the EO technique. The terms sign $(\vec{r}-0.5)$ have been responsible of the nearby path of explorations as well as exploitations. In $E O$, the values of $a_{1}$ and $a_{2}$ are elected to be 2 and 1 , respectively.

With replacing equation (23) in (21), the terms are altered as follows:

$$
\vec{F}=a_{1} \operatorname{sign}(\vec{r}-0.5)\left[e^{-\vec{\lambda} t}-1\right] .
$$

The generation rate in the EO technique was implemented for improving exploitation that is employed as a function of time. The $1^{\text {st }}$ order exponential decay procedure from the method of generation rate of a multipurpose technique is determined as follows:

$$
\vec{G}=\vec{G}_{0} e^{-\vec{k}\left(t-t_{0}\right)},
$$

where $G_{0}=$ primary value and $k=$ decay parameters.

Eventually, the generation rate appearance considering $k=\lambda$ has been determined as follows:

$$
\begin{aligned}
\vec{G} & =\vec{G}_{0} e^{-\vec{\lambda}\left(t-t_{0}\right)}, \\
& =\vec{G}_{0} \vec{F}_{0} .
\end{aligned}
$$

In equation (26), $G_{0}$ has been estimated as follows:

$$
\begin{aligned}
\vec{G}_{0} & =G \vec{C} P\left(\vec{C}_{e q}-\vec{\lambda} \vec{C}\right), \\
G \vec{C} P & = \begin{cases}0.5 r_{1}, & r_{2} \geq 0, \\
0, & r_{2}<0,\end{cases}
\end{aligned}
$$

where $r_{1}, r_{2}$ demonstrate 2 arbitrary numbers from intervals 0 and 1 and GCP denotes generation rate control parameter control generation rate. By means of all the abovementioned expressions, the last upgrade formula of concentration (particle) is determined as follows:

$$
\begin{gathered}
\vec{C}=\vec{C}_{e q}+\left(\vec{C}-\vec{C}_{e q}\right) \vec{F} \\
+\frac{\vec{G}}{\vec{\lambda} V}(1-\vec{F}) .
\end{gathered}
$$

The upgraded formula has 3 terms: 1 st term is equilibrium concentration; the 2 nd term is employed to global search; and 3rd term is in charge of local search for achieving exact solution.

3.2.3. Process Involved in the HS Algorithm. In HSA, the feasible solution (or element from the solution spaces) is called "harmony" that is an n-dimension real vector whose arbitrary values have been allocated to the primary population and loaded from harmony memory (HM). During the next step, an estimated novel candidate (nest generation/iteration) harmony was formed regarded as the element from HM, either by changing the pitch or by arbitrarily choosing to upgrade the element from $\mathrm{HM}$. As the last step, the element from the harmony memory is correlated with the least HM vector and recently calculated candidate harmony, and the entire procedure is repeated an amount of times for satisfying the end condition.

The HS optimization technique parameters are as follows: (i) size of HM, (ii) HM consideration rate (HMCR), (iii) pitch adjusting rate (PAR), and (iv) distance bandwidth (BW), and the number of iterations or improvisations (NI). During this step, it can be vital for configuring the primary HM modules (HMS vectors). Assume that $x i=\{x i(1), x i(2), \ldots x i(n)\}$ represents an arbitrarily calculated HM vector: $x i(k)=X_{l}(k)+\left(X_{u}(k)-X_{l}(k)\right) *$ rand $(0,1)$ for $k=$ $1,2, \ldots$, nandi $=1,2, \ldots, H M S$ which is the length of HM. So, the lower as well as upper restricts of feasible search space are represented as $X_{l}(k)$ and $X_{u}(k)$, respectively [25]. Next, in the HM matrix, all components are a harmony vector, so it can be that HMS has a vector present.

$$
H M=\left[\begin{array}{c}
x_{1} \\
x_{2} \\
\vdots \\
x_{H M S}
\end{array}\right] .
$$

In the HM matrix, $x_{\text {new }}$, a novel harmony vector, has been created utilizing 3 functions: (i) memory consideration, (ii) arbitrary reinitialization, and (iii) pitch adjustment. During the initial stage, the primary decision value $x_{\text {new }}(1)$ was chosen arbitrarily in harmony provided as $\left\{x_{1}(1), x_{2}(1) \ldots x_{H M S}(1)\right\}$. In order to choose $x_{\text {new }}(1)$ an arbitrary number $r 1$ with a range of 0 and 1 , when this arbitrary number has been under HMCR, $x_{\text {new }}(1)$ has been created as a memory consideration, otherwise $x_{\text {new }}(1)$ has been captured in the search range $\left[X_{l}(k), X_{u}(k)\right]$. In the same manner, $x_{\text {new }}(2), x_{\text {new }}(3), \ldots \ldots, x_{\text {new }}(n)$ are chosen so that the 2 functions such as memory consideration and arbitrary reinitialization are determined as follows: 


$$
x_{\text {new }}(k)=\left\{\begin{array}{l}
x_{i} \in\left\{x_{1}(k) \ldots \ldots \ldots \ldots x_{H M S}(k)\right\}, \\
X_{l}(k)+\left(X_{u}(k)-X_{l}(k)\right) * \operatorname{ran} d(0,1), \\
\text { with probability of 1-HMCR. }
\end{array}\right.
$$

All recently created $x_{\text {new }}$ values were examined to determine whether they can be pitch adjusted or not. To resolve this, the pitch adjustment rate (PAR) has been developed which is a composition of frequency and bandwidth factor (BF). These 2 factors are modified to obtain a novel $x_{\text {new }}$ value in local search for the chosen solutions of HM. The pitch modified novel solution $x_{\text {new }}$ has been computed as $x_{\text {new }}(k)+/-\operatorname{rand}(0,1) \cdot B W$ with probabilities PAR. This pitch adjustment is most same as the mutation procedure used in some evolutionary bioinspired techniques. The range of pitch adjustment has been restricted as $\left[X_{l}(k), X_{u}(k)\right]$.

From the last phase, $x_{\text {new }}$, a novel harmony vector created, the HM has been estimated/upgraded as a novel completion to fitness amongst $x_{\text {new }}$ and a worse harmony vector $x_{w}$ in the HM. Accordingly $x_{w}$ has been changed by $x_{\text {new }}$ and developed as part of HM. To maximize the objective function (interclass variance), HSA has been utilized.

The harmony or solution utilizes $k$ distinct elements for deciding the optimized technique. These variables have been threshold values $t h_{k}$ which is more utilized to multilevel segmentation. The population of the technique has been expressed as follows:

$$
\begin{aligned}
H M & =\left[x_{1}, x_{2} \ldots, x_{1 H M S}\right]^{T}, \\
x_{i} & =\left[t h_{1}, t h_{2} \ldots . t h_{k}\right] .
\end{aligned}
$$

In the abovementioned formula $T$ represents the transpose of the matrix, the maximal size of HM has been represented as HMS, and all the elements from HM have been demonstrated as $x i$, where the range of $i$ is $[0, k]$. Figure 3 demonstrates the flowchart of the HS technique.

\section{Experimental Validation}

Table 1 provides the results analysis of the SEF with three optimization algorithms under five distinct runs. Figure 4 showcases the results analysis of the SEF-CS technique on five test runs. The results demonstrated that the SEF-CS technique has accomplished maximum performance under all runs. For instance, with run-1, the SEF-CS technique has attained a dice coefficient of $84.89 \%$, an IoU of $73.92 \%$, a sensitivity of $96.76 \%$, and an accuracy of $98.38 \%$, respectively. In addition, under run-3, the SEF-CS technique has offered a dice coefficient of $83.52 \%$, an IoU of $72.32 \%$, a sensitivity of $95.26 \%$, and an accuracy of $98.07 \%$, respectively. Moreover, the SEF-CS technique has provided an average dice coefficient of $84.92 \%$, an IoU of $73.82 \%$, a sensitivity of $96.66 \%$, and an accuracy of $98.46 \%$, respectively.

Figure 5 illustrates the results analysis of the SEF-EO technique on five test runs. The results demonstrated that the SEF-EO technique has accomplished maximum performance under all runs. For instance, with run-1, the SEF-EO technique has attained a dice coefficient of $83.77 \%$, an IoU of
$71.75 \%$, a sensitivity of $95.26 \%$, and an accuracy of $98.09 \%$, respectively. Besides, under run-3, the SEF-EO approach has offered a dice coefficient of $84.93 \%$, an IoU of $73.55 \%$, a sensitivity of $96.87 \%$, and an accuracy of $98.49 \%$, respectively. Furthermore, the SEF-EO technique has provided an average dice coefficient of $85.44 \%$, an IoU of $73.39 \%$, a sensitivity of $96.76 \%$, and an accuracy of $98.55 \%$, correspondingly.

Figure 6 depicts the results analysis of the SEF-HS technique on five test runs. The results demonstrated that the SEF-HS technique has accomplished maximum performance under all runs. For instance, with run-1, the SEF-HS technique has attained a dice coefficient of $83.59 \%$, an IoU of $72.25 \%$, a sensitivity of $95.65 \%$, and an accuracy of $98.18 \%$, respectively. At the same time, under run-3, the SEF-HS technique has offered a dice coefficient of $85.45 \%$, an IoU of $73.55 \%$, a sensitivity of $97.55 \%$, and an accuracy of $98.69 \%$, respectively. Finally, the SEF-HS technique has provided an average dice coefficient of $85.27 \%$, an IoU of $73.69 \%$, a sensitivity of $97.23 \%$, and an accuracy of $98.62 \%$, correspondingly.

Table 2 provides the results analysis of the MLOT with three optimization techniques under five distinct runs. Figure 7 demonstrates the results analysis of the MLOT-CS technique on five test runs. The results demonstrated that the MLOT-CS technique has accomplished maximum performance under all runs. For instance, with run-1, the MLOTCS technique has attained a dice coefficient of $85.31 \%$, an IoU of $72.25 \%$, a sensitivity of $95.80 \%$, and an accuracy of $98.25 \%$, respectively. Likewise, under run-3, the MLOT-CS technique has offered a dice coefficient of $86.51 \%$, an IoU of $73.82 \%$, a sensitivity of $97.60 \%$, and an accuracy of $98.55 \%$, respectively. Eventually, the MLOT-CS technique has provided an average dice coefficient of $86.55 \%$, an IoU of $73.83 \%$, a sensitivity of $97.44 \%$, and an accuracy of $98.67 \%$, respectively.

Figure 8 showcases the results analysis of the MLOT-EO technique on five test runs. The results demonstrated that the MLOT-EO technique has accomplished maximum performance under all runs. For instance, with run-1, the MLOTEO technique has attained a dice coefficient of $85.31 \%$, an IoU of $72.25 \%$, a sensitivity of $96.15 \%$, and an accuracy of $98 \%$, respectively. Moreover, under run-3, the MLOT-EO technique has offered a dice coefficient of $87.21 \%$, an IoU of $78.85 \%$, a sensitivity of $97.65 \%$, and an accuracy of $98.18 \%$, respectively. Then, the MLOT-EO technique provided an average dice coefficient of $87.07 \%$, an IoU of $73.97 \%$, a sensitivity of $97.63 \%$, and an accuracy of $98.64 \%$, correspondingly.

Figure 9 exhibits the results analysis of the MLOT-HS technique on five test runs. The results demonstrated that the MLOT-HS technique has accomplished maximum performance under all runs. For instance, with run-1, the MLOTHS technique has attained a dice coefficient of $85.41 \%$, an IoU of $72.60 \%$, a sensitivity of $96.97 \%$, and an accuracy of $98.01 \%$, respectively. Followed by, under run-3, the MLOTHS technique has offered a dice coefficient of $86.91 \%$, an IoU of $74.30 \%$, a sensitivity of $98.12 \%$, and an accuracy of $98.90 \%$, respectively. Finally, the MLOT-HS technique has provided 


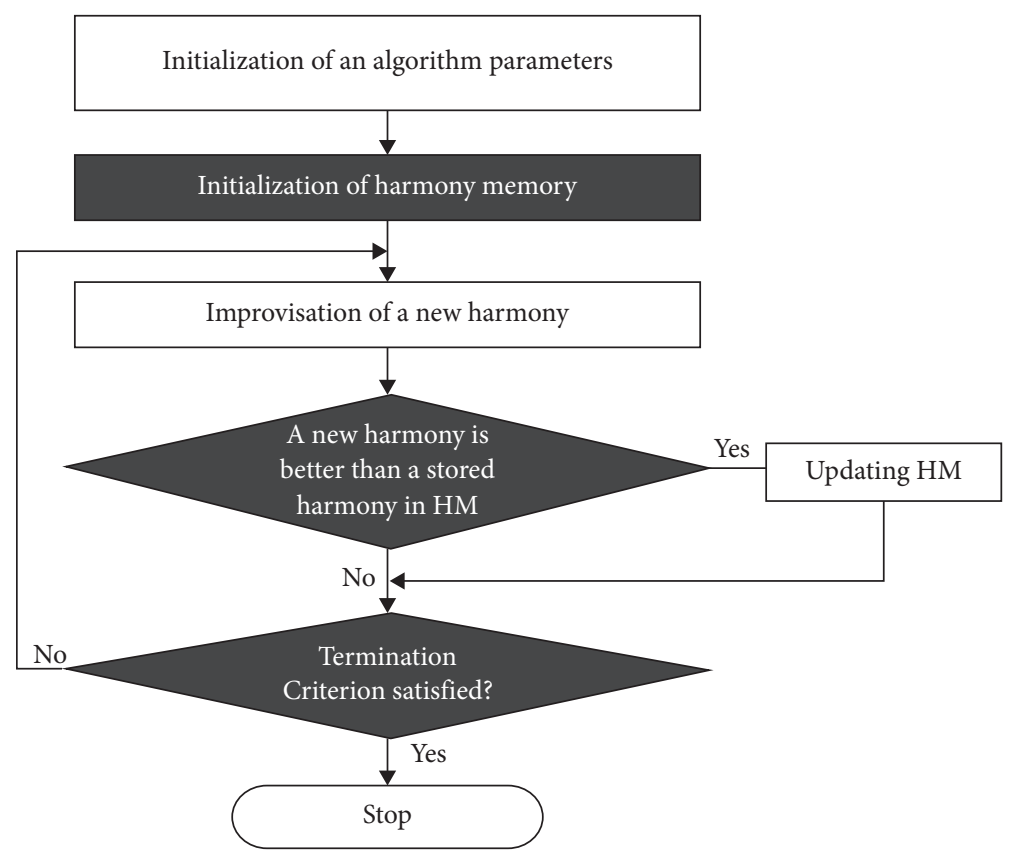

FIGURE 3: Flowchart of the HS algorithm.

TABLE 1: Result analysis of SEF with three optimization algorithms

\begin{tabular}{lcccc}
\hline No. of runs & Dice coefficient & IoU & Sensitivity & Accuracy \\
\hline SEF-CS & & & & \\
Run-1 & 83.52 & 72.32 & 95.26 & 98.07 \\
Run-2 & 84.37 & 72.92 & 96.26 & 98.28 \\
Run-3 & 84.89 & 73.92 & 96.76 & 98.38 \\
Run-4 & 85.54 & 74.52 & 97.26 & 98.68 \\
Run-5 & 86.29 & 75.42 & 97.76 & 98.89 \\
Average & 84.92 & 73.82 & 96.66 & 98.46 \\
\hline SEF-EO & & & & \\
Run-1 & 83.77 & 71.75 & 95.26 & 98.09 \\
Run-2 & 84.34 & 72.65 & 96.04 & 98.15 \\
Run-3 & 84.93 & 73.55 & 96.87 & 98.49 \\
Run-4 & 85.72 & 74.15 & 97.41 & 98.88 \\
Run-5 & 86.44 & 74.85 & 98.20 & 99.15 \\
Average & 85.04 & 73.39 & 96.76 & 98.55 \\
\hline SEF-HS & & & & \\
Run-1 & 83.59 & 72.25 & 95.65 & 98.18 \\
Run-2 & 84.52 & 72.85 & 96.85 & 98.35 \\
Run-3 & 85.45 & 73.55 & 97.55 & 98.69 \\
Run-4 & 86.17 & 74.45 & 97.95 & 98.88 \\
Run-5 & 86.64 & 75.35 & 98.15 & 99.02 \\
Average & 85.27 & 73.69 & 97.23 & 98.62 \\
\hline
\end{tabular}

an average dice coefficient of $87.09 \%$, an IoU of $74.30 \%$, a sensitivity of $98.22 \%$, and an accuracy of $98.71 \%$, correspondingly.

Finally, a comprehensive comparison study of the proposed and existing techniques takes place in Table 3. Figure 10 investigates the dice coefficient analysis of the proposed with existing techniques. The results show that the maximum entropy, region growing, DCNN-FCN, thresholding segmentation, and K-means clustering techniques have accomplished ineffective outcomes compared to the other techniques. At the same time, the DCNN-UNet,

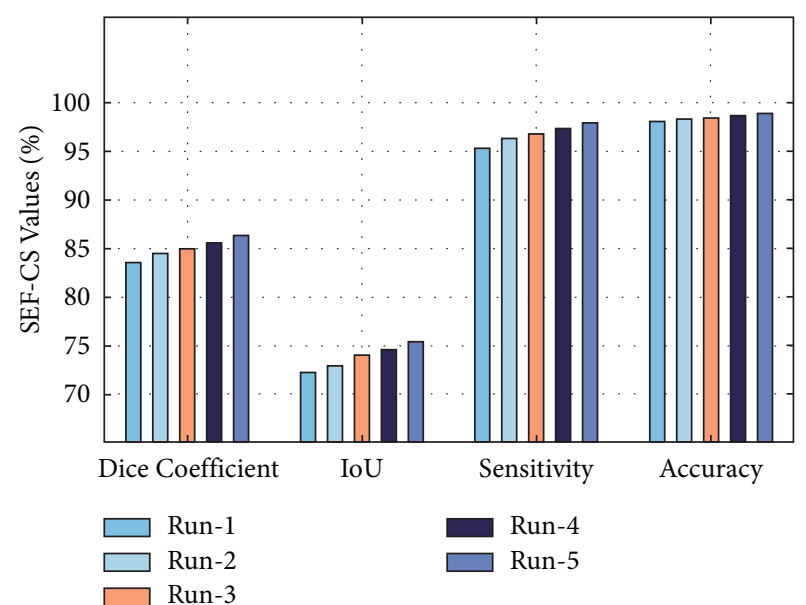

FIgUre 4: Result analysis of the SEF-CS model with different runs.

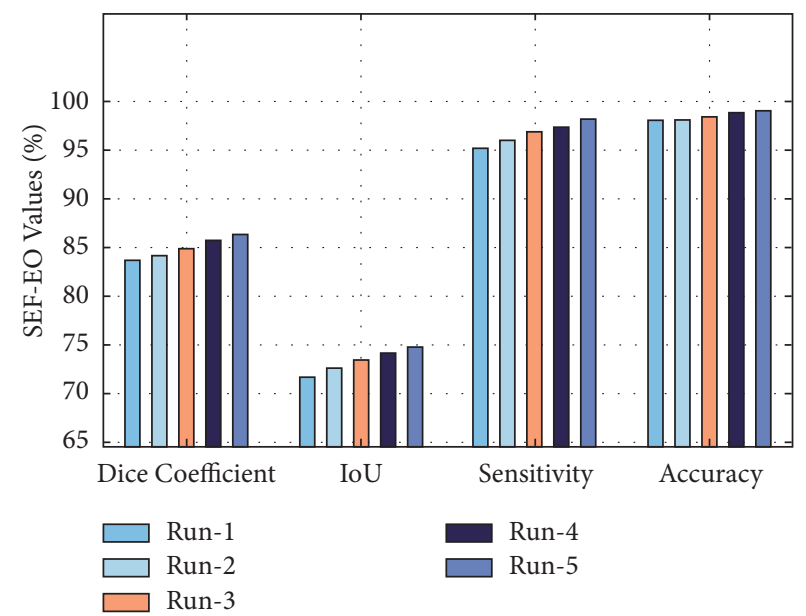

FIgURE 5: Result analysis of the SEF-EO model with different runs. 


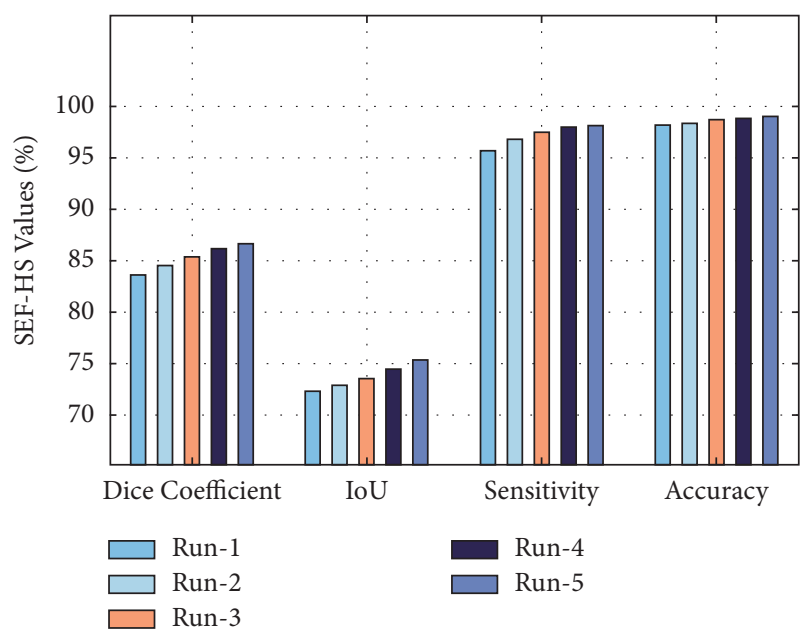

FIGURE 6: Result analysis of the SEF-HS model with different runs.

TABLE 2: Result analysis of MLOT with three optimization algorithms.

\begin{tabular}{lcccc}
\hline No. of runs & Dice coefficient & IoU & Sensitivity & Accuracy \\
\hline MLOT-CS & & & & \\
Run-1 & 85.31 & 72.25 & 95.80 & 98.25 \\
Run-2 & 86.01 & 72.93 & 96.70 & 98.45 \\
Run-3 & 86.51 & 73.82 & 97.60 & 98.55 \\
Run-4 & 87.11 & 74.81 & 98.20 & 98.95 \\
Run-5 & 87.81 & 75.35 & 98.90 & 99.16 \\
Average & 86.55 & 73.83 & 97.44 & 98.67 \\
\hline MLOT-EO & & & & \\
Run-1 & 85.31 & 72.25 & 96.15 & 98.00 \\
Run-2 & 86.21 & 73.25 & 96.95 & 98.18 \\
Run-3 & 87.21 & 73.85 & 97.65 & 98.78 \\
Run-4 & 87.91 & 74.85 & 98.45 & 99.10 \\
Run-5 & 88.71 & 75.65 & 98.96 & 99.14 \\
Average & 87.07 & 73.97 & 97.63 & 98.64 \\
\hline MLOT-HS & & & & \\
Run-1 & 85.41 & 72.60 & 96.97 & 98.01 \\
Run-2 & 86.41 & 73.50 & 97.73 & 98.54 \\
Run-3 & 86.91 & 74.30 & 98.12 & 98.90 \\
Run-4 & 87.91 & 75.30 & 99.05 & 98.97 \\
Run-5 & 88.81 & 75.80 & 99.25 & 99.13 \\
Average & 87.09 & 74.30 & 98.22 & 98.71 \\
\hline
\end{tabular}

DCNN-Hy-Net, SEF-CS, SEF-EO, and SEF-HS techniques have accomplished moderately closer dice coefficient values. Followed by the MLOT-CS and MLOT-EO techniques, they have resulted in certainly improved dice coefficient values of $86.55 \%$ and $87.07 \%$, respectively. However, the MLOT-HS technique has gained effective performance with a higher dice coefficient of $87.09 \%$.

Figure 11 examines the IoU analysis of the proposed with existing techniques. The results show that the maximum entropy, region growing, DCNN-FCN, thresholding segmentation, and K-means clustering techniques have accomplished ineffective outcomes compared to the other techniques. In line with the DCNN-UNet, DCNN-Hy-Net, SEF-CS, SEF-EO, and SEF-HS techniques, they have accomplished moderately closer IoU values. Following that the MLOT-CS and MLOT-EO techniques have resulted to

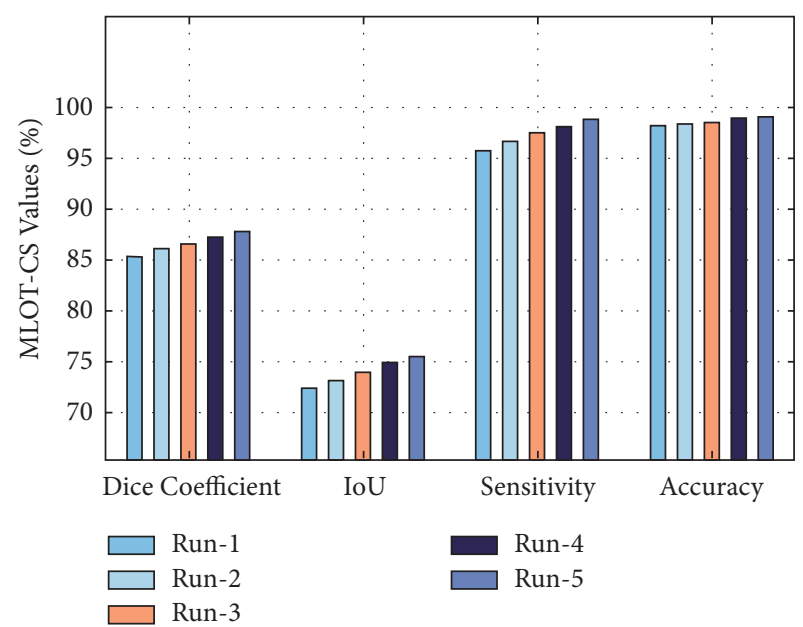

Figure 7: Result analysis of the MLOT-CS model with different runs.

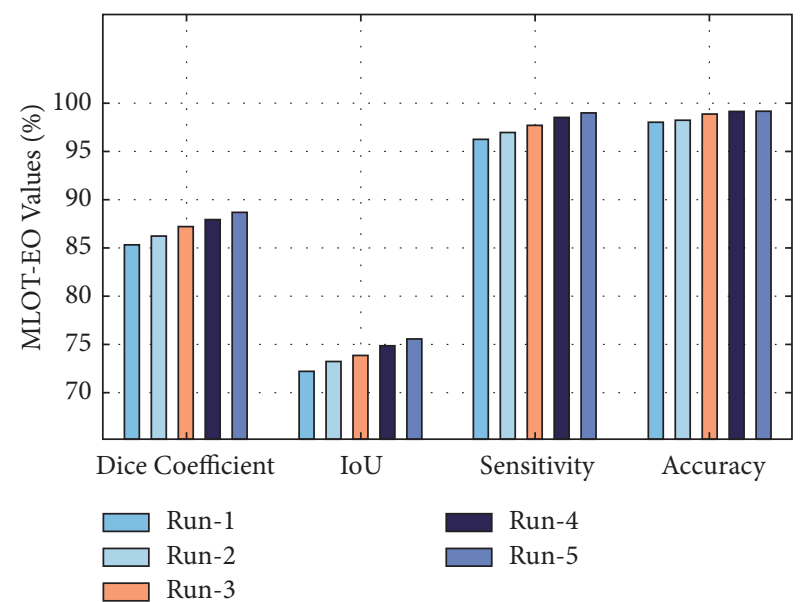

FIgUre 8: Result analysis of the MLOT-EO model with different runs.

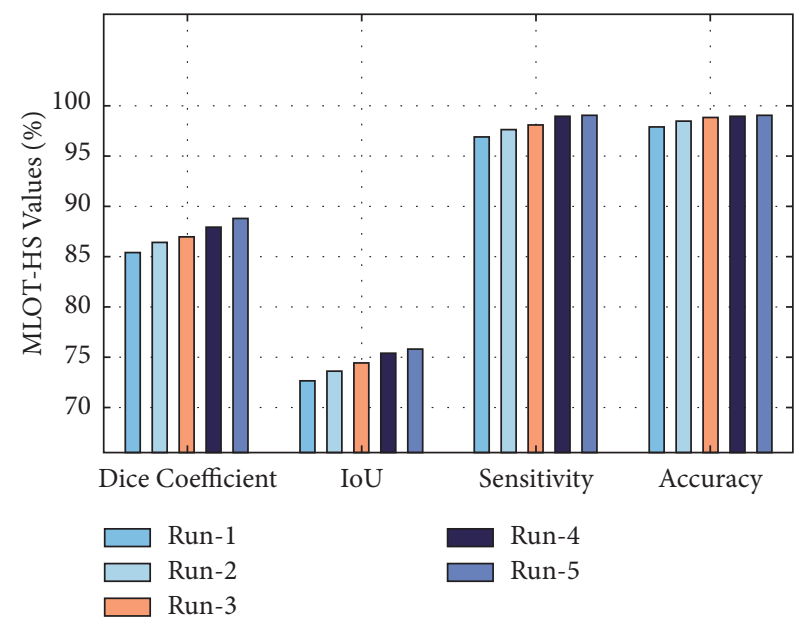

FIgURE 9: Result analysis of the MLOT-HS model with different runs. 
TABLE 3: Comparative results analysis of proposed versus existing techniques.

\begin{tabular}{lcccc}
\hline Methods & $\begin{array}{c}\text { Dice } \\
\text { coefficient }\end{array}$ & IoU & Sensitivity & Accuracy \\
\hline Threshold & 71.18 & 56.49 & 61.74 & 97.80 \\
segmentation & 64.80 & 49.57 & 52.36 & 97.46 \\
Region growing & 50.87 & 35.53 & 95.85 & 91.22 \\
Maximum entropy & 75.51 & 61.13 & 71.22 & 97.99 \\
K-means clustering & 68.67 & 52.40 & 70.62 & 96.83 \\
DCNN-FCN & 82.38 & 70.37 & 83.89 & 98.09 \\
DCNN-UNet & 83.91 & 72.65 & 89.14 & 98.28 \\
DCNN-Hy-net & 84.92 & 73.82 & 96.66 & 98.46 \\
SEF-CS & 85.04 & 73.39 & 96.76 & 98.55 \\
SEF-EO & 85.27 & 73.69 & 97.23 & 98.62 \\
SEF-HS & 86.55 & 73.83 & 97.44 & 98.67 \\
MLOT-CS & 87.07 & 73.97 & 97.63 & 98.64 \\
MLOT-EO & 87.09 & 74.30 & 98.22 & 98.71 \\
MLOT-HS & & & & \\
\hline
\end{tabular}

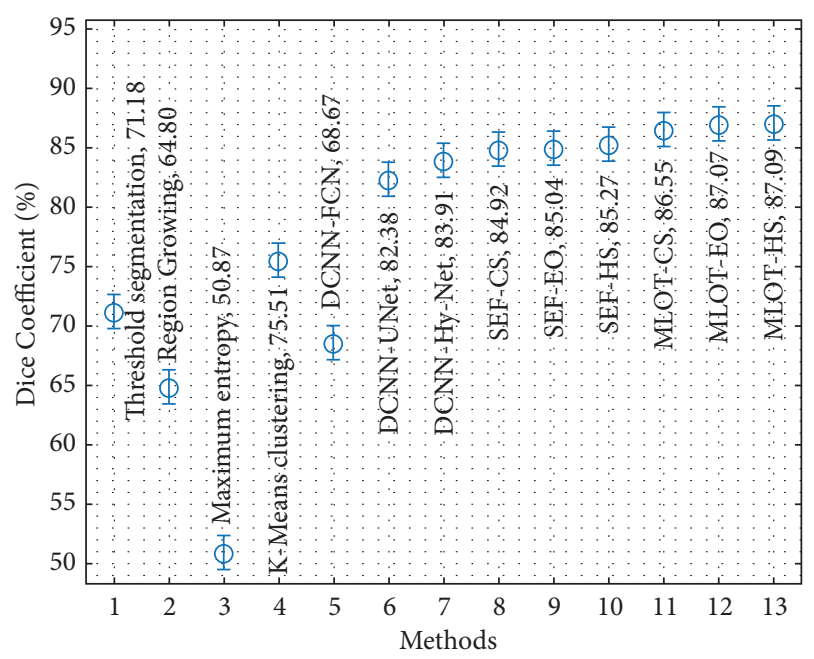

FIgURE 10: Comparative analysis of proposed method interms of dice coefficient.

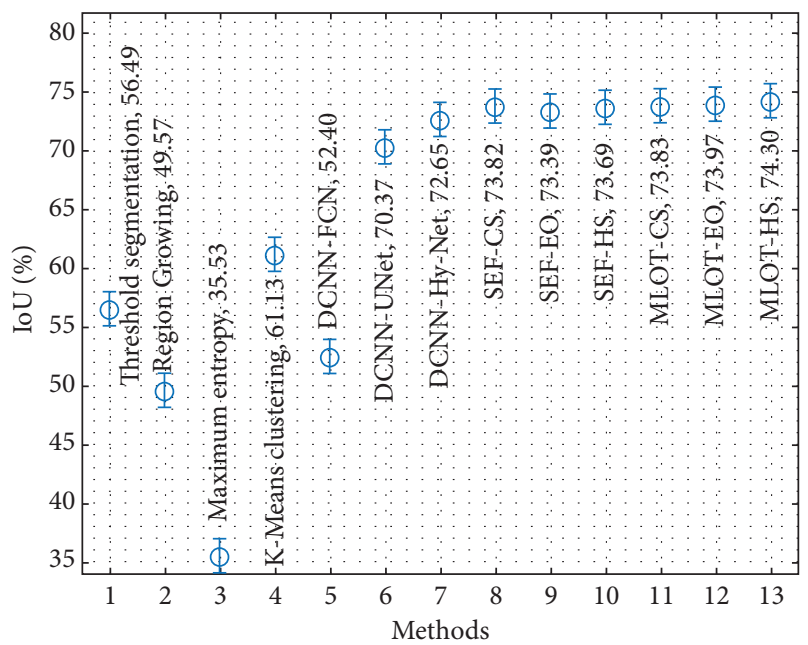

FIGURE 11: Comparative analysis of the proposed method in terms of IoU.

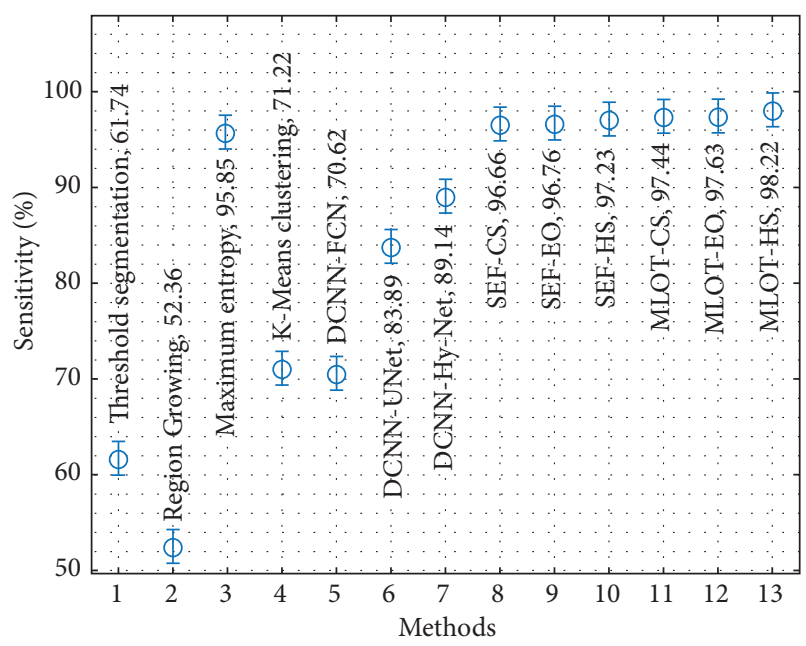

Figure 12: Comparative analysis of the proposed method in terms of sensitivity.

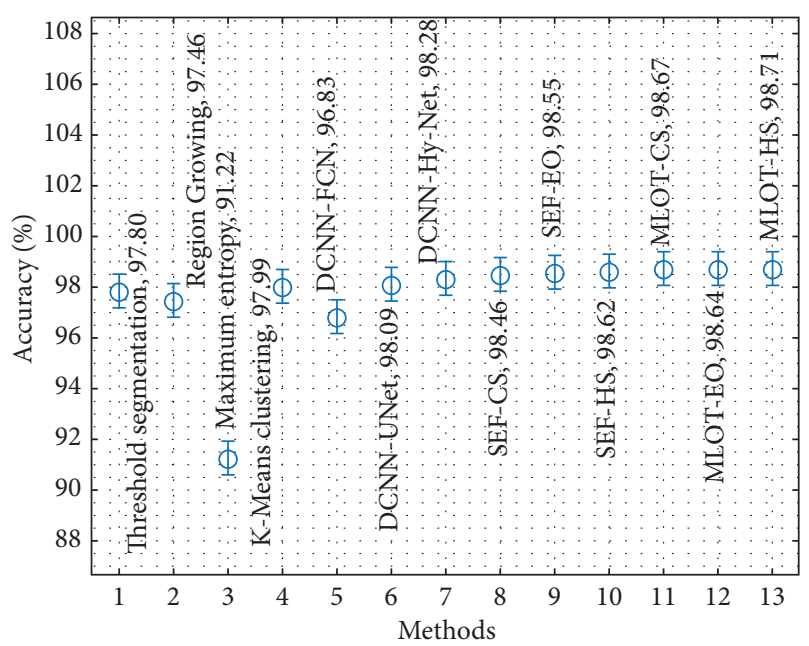

FIGURE 13: Comparative analysis of the proposed method in terms of accuracy.

certainly improved IoU values of $73.83 \%$ and $73.97 \%$, correspondingly. However, the MLOT-HS technique has gained effective performance with a higher IoU of $74.30 \%$.

Figure 12 considers the sensitivity analysis of the proposed with existing techniques. The results show that the maximum entropy, region growing, DCNN-FCN, thresholding segmentation, and $K$-means clustering techniques have accomplished ineffective outcomes compared to the other techniques. Simultaneously, the DCNN-UNet, DCNN-Hy-Net, SEF-CS, SEF-EO, and SEF-HS techniques have accomplished moderately closer sensitivity values. Also, the MLOT-CS and MLOT-EO techniques have resulted to certainly improved sensitivity values of $97.44 \%$ and $97.63 \%$, respectively. However, the MLOT-HS technique has gained effective performance with a higher sensitivity of $98.22 \%$.

Figure 13 explores the accuracy analysis of the proposed with existing techniques. The results show that the maximum entropy, region growing, DCNN-FCN, thresholding 
segmentation, and $K$-means clustering techniques have accomplished ineffective outcomes compared to the other techniques. Concurrently, the DCNN-UNet, DCNN-HyNet, SEF-CS, SEF-EO, and SEF-HS techniques have accomplished moderately closer accuracy values. Similarly, the MLOT-CS and MLOT-EO techniques have resulted to certainly improved accuracy values of $98.67 \%$ and $98.64 \%$, respectively. Lastly, the MLOT-HS technique has gained effective performance with a higher accuracy of $98.71 \%$. By looking into the abovementioned tables and figures, it is ensured that the MLOT-HS technique is found to be effective compared to other techniques.

\section{Conclusion}

In this study, a set of metaheuristic optimization-based vascular segmentation techniques have been developed for PAI. The goal of the study is to examine the outcomes of the different metaheuristics on two segmentation approaches. In order to effectively segment the vessels, the SEF and MLOT techniques are applied. Moreover, three optimization algorithms such as CS, EO, and HS are utilized for optimal selection of entropy function and threshold values. For examining the improved performance of the proposed model, a wide range of simulations take place and the results are inspected under various aspects. The experimental results pointed out that the proposed MLOT-HS technique has gained maximum performance over the other existing approaches. Thus, it can be employed in real-time brain imaging related applications such as brain and cancer imaging such as prostate cancer, breast cancer, and ovarian cancer. In the future, the vessel segmentation performance of the proposed model can be improved by the use of deep learning models.

\section{Data Availability}

No datasets were generated during this study.

\section{Ethical Approval}

This article does not contain any studies with human participants performed by any of the authors.

\section{Conflicts of Interest}

The authors declare that they have no conflicts of interest.

\section{Authors' Contributions}

The manuscript was written by contributions from all authors. All authors have given approval to the final version of the manuscript.

\section{Acknowledgments}

This research was supported by Taif University Researchers Supporting Project (number TURSP-2020/314), Taif University, Taif, Saudi Arabia.

\section{References}

[1] C. D. Ly, V. T. Nguyen, T. H. Vo et al., "Full-view in vivo skin and blood vessels profile segmentation in photoacoustic imaging based on deep learning," Photoacoustics, vol. 25, Article ID 100310, 2022.

[2] Y. Zhou, J. Yao, and L. V. Wang, "Tutorial on photoacoustic tomography," Journal of Biomedical Optics, vol. 21, no. 6, Article ID 61007, 2016.

[3] H. Zhong, T. Duan, H. Lan, M. Zhou, and F. Gao, "Review of low-cost photoacoustic sensing and imaging based on laser diode and light-emitting diode," Sensors Basel (Basel), vol. 18, no. 7, 2018

[4] H. Lan, T. Duan, H. Zhong, M. Zhou, and F. Gao, "Photoacoustic classification of tumor model morphology based on support vector machine: a simulation and phantom study," IEEE Journal of Selected Topics in Quantum Electronics, vol. 25, no. 1, pp. 1-9, 2019.

[5] X. Zhang, F. Ma, Y. Zhang, J. Wang, C. Liu, and J. Meng, "Sparse-sampling photoacoustic computed tomography: deep learning vs. compressed sensing," Biomedical Signal Processing and Control, vol. 71, Article ID 103233, 2022.

[6] T. Duan, H. Lan, H. Zhong, M. Zhou, R. Zhang, and F. Gao, "Hybrid multi-wavelength nonlinear photoacoustic sensing and imaging," Optics Letters, vol. 43, no. 22, pp. 5611-5614, 2018.

[7] L. Lin, P. Hu, J. Shi et al., "Singlebreath-hold photoacoustic computed tomography of the breast," Nature Communications, vol. 9, no. 1, p. 2352, 2018.

[8] Y. E. Boink, S. Manohar, and C. Brune, "A partially-learned algorithm for joint photo-acoustic reconstruction and segmentation," IEEE Transactions on Medical Imaging, vol. 39, no. 1, pp. 129-139, 2020.

[9] S. Hu and L. Wang, "Optical-resolution photoacoustic microscopy: auscultation of biological systems at the cellular level," Biophysical Journal, vol. 105, no. 4, pp. 841-847, 2013.

[10] E. H. Houssein, B. E. D. Helmy, D. Oliva, A. A. Elngar, and H. Shaban, "A novel Black Widow Optimization algorithm for multilevel thresholding image segmentation," Expert Systems with Applications, vol. 167, Article ID 114159, 2021.

[11] E. H. Houssein, B. E. D. Helmy, A. A. Elngar, D. S. Abdelminaam, and H. Shaban, "An improved tunicate swarm algorithm for global optimization and image segmentation," IEEE Access, vol. 9, pp. 56066-56092, 2021.

[12] E. H. Houssein, M. M. Emam, and A. A. Ali, "An efficient multilevel thresholding segmentation method for thermography breast cancer imaging based on improved chimp optimization algorithm," Expert Systems with Applications, vol. 185, Article ID 115651, 2021.

[13] G. P. Luke, K. Hoffer-Hawlik, A. C. Van Namen, and R. Shang, "O-Net: a convolutional neural network for quantitative photoacoustic image segmentation and oximetry," 2019, https://arxiv.org/abs/1911.01935.

[14] C. Bench, A. Hauptmann, and B. T. Cox, "Toward accurate quantitative photoacoustic imaging: learning vascular blood oxygen saturation in three dimensions," Journal of Biomedical Optics, vol. 25, no. 8, Article ID 085003, 2020.

[15] P. Raumonen and T. Tarvainen, "Segmentation of vessel structures from photoacoustic images with reliability assessment," Biomedical Optics Express, vol. 9, no. 7, pp. 2887-2904, 2018.

[16] A. Y. Yuan, Y. Gao, L. Peng et al., "Hybrid deep learning network for vascular segmentation in photoacoustic imaging," Biomedical Optics Express, vol. 11, no. 11, pp. 6445-6457, 2020. 
[17] M. Sun, C. Li, N. Chen et al., "Full three-dimensional segmentation and quantification of tumor vessels for photoacoustic images," Photoacoustics, vol. 20, Article ID 100212, 2020.

[18] J. Zhao, Q. Zhao, R. Lin, and J. Meng, "A microvascular image analysis method for optical-resolution photoacoustic microscopy," Journal of Innovative Optical Health Sciences, vol. 13, no. 04, Article ID 2050019, 2020.

[19] Y. E. Boink, S. Manohar, and C. Brune, "A partially-learned algorithm for joint photo-acoustic reconstruction and segmentation," IEEE Transactions on Medical Imaging, vol. 39, no. 1, pp. 129-139, 2019.

[20] T. Feng, Y. Zhu, C. Liu et al., "Ultrasound-guided detection and segmentation of photoacoustic signals from bone tissue in vivo," Applied Sciences, vol. 11, no. 1, p. 19, 2021.

[21] D. K. Patra, T. Si, S. Mondal, and P. Mukherjee, "Breast DCEMRI segmentation for lesion detection by multi-level thresholding using student psychological based optimization," Biomedical Signal Processing and Control, vol. 69, Article ID 102925, 2021.

[22] N. Otsu, "A threshold selection method from gray-level histograms," IEEE Transactions on Systems, Man, and Cybernetics, vol. 9, no. 1, pp. 62-66, 1979.

[23] J. Rahaman and M. Sing, "An efficient multilevel thresholding based satellite image segmentation approach using a new adaptive cuckoo search algorithm," Expert Systems with Applications, vol. 174, Article ID 114633, 2021.

[24] S. K. Dinkar, K. Deep, S. Mirjalili, and S. Thapliyal, "Opposition-based laplacian equilibrium optimizer with application in image segmentation using multilevel thresholding," Expert Systems with Applications, vol. 174, Article ID 114766, 2021.

[25] R. Srikanth and K. Bikshalu, "Multilevel thresholding image segmentation based on energy curve with harmony Search Algorithm," Ain Shams Engineering Journal, vol. 12, no. 1, pp. 1-20, 2021. 